\title{
Observation of the microwave near-field enhancement effect in suspensions comprising single-walled carbon nanotubes
}

\author{
M. V. Shuba ${ }^{1}$, A. G. Paddubskaya ${ }^{2}$, P. P. Kuzhir ${ }^{1}$, S. A. \\ Maksimenko ${ }^{1}$, G. Valusis ${ }^{2,3}$, M. Ivanov ${ }^{3}$, J. Banys ${ }^{3}$, V. \\ Ksenevich $^{4}$, and G. W. Hanson ${ }^{5}$ \\ ${ }^{1}$ Institute for Nuclear Problems, Belarus State University, Bobruiskaya 11, 220030 \\ Minsk, Belarus \\ 2 Center for Physical Sciences and Technology, Sauletekio st. 3, Vilnius LT-10222, \\ Lithuania \\ ${ }^{3}$ Faculty of Physics, Vilnius University, Sauletekio 9/3, Vilnius LT-10222, Lithuania \\ 4 Department of Physics, Belarus State University, Nezalezhnastsi Av. 4, 220030 \\ Minsk, Belarus \\ ${ }^{5}$ Department of Electrical Engineering, University of Wisconsin-Milwaukee, \\ Milwaukee, Wisconsin 53211, USA \\ E-mail: mikhail.shuba@gmail.com
}

December 2015

\begin{abstract}
The strong polarization of isolated finite-length single-walled carbon nanotubes (CNTs) or bundles of CNTs has been predicted to lead to a strong near field enhancement effect that provokes additional electromagnetic energy dissipation in a conductive host [Shuba et al., J. Appl. Phys. 108, 114302 (2010)]. Here, we theoretically and experimentally show that this effect can be observed in the microwave frequency range via measurements of the spectra of the effective permittivity of CNTbased suspensions. Microwave effective permittivity of the suspensions comprising both annealed and doped CNTs has been measured in the temperature range from 300 to $330 \mathrm{~K}$. The experimental data give evidence of the unique properties of CNTs - to enhance electromagnetic fields in a volume as much as 100 times larger than the volume occupied by the nanotube itself.
\end{abstract}

PACS numbers: 78.70.Gq,73.63.Fg,77.22.Ej,73.20.Mf

Keywords: Near-field enhancement effect, suspension, single-walled carbon nanotube, microwave frequency range 


\section{Introduction}

A single-walled carbon nanotube (CNT) can be considered as a graphene sheet rolled up into a cylinder. Depending on the direction of rolling, the tube can be metallic or semiconducting [1]. Due to its elongated shape and high axial surface conductivity, finite-length metallic CNTs exposed to an external electromagnetic (EM) field can collect significant unlike charges on its opposite ends [2]. As the nanotube diameter is very small $(0.7-2 \mathrm{~nm})$, the surface charge density near the ends is large, and the radial component $E_{\rho}$ of the electric field vector induced by these charges is many times higher than the axial component of incident field $E_{0}$. This radial component $E_{\rho}$ decreases slowly with increasing distance $\rho$ from the nanotube axis. As a result, electromagnetic energy concentrates in a spacial volume which is significantly larger than the volume occupied by the CNT itself.

The effect of near-field enhancement does not relate to any resonance phenomena, and it occurs in the quasi-electrostatic regime, where the real part of the nanotube polarizability has a value close to the dc-polarizability. For a typical nanotube length of $1 \mu \mathrm{m}$, the quasi-electrostatic regime occurs at frequencies below $100 \mathrm{GHz}$. Due to the localized plasmon resonance in CNTs [3] in the range $0.1-10 \mathrm{THz}$, the near-field enhancement effect can be even stronger than in the quasi-electrostatic regime $[4,5]$.

Since its theoretical prediction, the near-field enhancement effect has been observed in the infrared range $(12-30 \mathrm{THz})$ via absorption enhancement of biomolecules absorbed on single-walled carbon nanotubes [6]. The aim of the present work is to specify conditions for the near-field enhancement effect in the microwave range.

In spite of the effective interaction of CNTs with EM radiation $[2,7,8]$, the electromagnetic response of a single CNT is too small to be detected by modern experimental techniques [9]. Therefore, in practice, CNT ensembles or networks are frequently under investigation in the microwave and terahertz ranges [9, 10]. The EM parameters of CNT suspensions without agglomerates should have a simple relationship with the EM response of individual CNTs [11, 12, 13], as effects of both electromagnetic and electronic intertube interactions are very small and can be neglected in this case. Therefore, CNT suspensions can be considered as an effective tool for investigation of EM parameters of individual CNTs from the radiofrequency to terahertz range [10].

Currently there are several obstacles which prevent the wide application of microwave spectroscopy to the study of CNTs: (i) only small CNT densities $(<1$ $\mathrm{mg} / \mathrm{ml}$ ) can be achieved in well-dispersed suspensions [14] resulting in small differences between the electromagnetic response of liquid media with and without CNTs $[15,16$, 17, 18, 19, 20]; (ii) dispersion of CNTs in the solvent is accompanied by CNT length reduction [21]; and (iii) at present there is a lack of understanding and difficulties in the interpretation of the data from microwave measurements.

Another obstacle is that, in practice, carbon nanotube materials comprise CNTs with different conductivities. The materials commercially available for enriched metallic or semiconducting nanotubes contain some residue of iodixanol or other chemicals that 
can not be completely removed from the tube surfaces after separation [22]. As a rule, the purification and separation processes lead to CNT doping, so that even highly enriched semiconducting CNT films have conductivity comparable to that of highly enriched metallic CNT films [23]. An annealing process can be used to partly remove the doping effect and decrease by several times the CNT film conductivity, but it does not solve the problem completely - after annealing atmospheric oxygen attaches to the sidewall defects at the CNT surface, providing a slight unintentional p-type doping effect [24], so that enriched semiconducting CNTs, after annealing, still demonstrate metallic behavior (see Figs. 2b and 2c in [25]). Moreover, annealing strengthens the adhesion of the tubes inside agglomerates, which prevents their exfoliation and dispersion in liquid media.

Although, as described above, even semiconducting CNTs have moderately-high conductivity due to unintentional doping, in the following, for generality, we consider the possibility to have both low- and high-conductivity CNTs.

In order to demonstrate how low- and high-conductive tubes can influence the effective microwave parameters of CNT-based suspensions, we apply an homogenization model to idealized suspensions comprised of a mixture of individual low-conductive semiconducting (s-CNTs) and high-conductive metallic (m-CNTs) tubes. And, we carry out a microwave spectroscopy study of CNT-based suspensions. The experimental data and theoretical analysis give evidence of the effect of the microwave absorption enhancement due to near-field enhancement in CNTs.

\section{Theoretical consideration}

\subsection{Near-field enhancement effect in the vicinity of CNTs}

Using the theory of electromagnetic wave scattering by a finite length CNT (for details see Supplemental Material [26] and Refs. [11, 13, 4]), we calculated the electric field $E(\rho, z)$ scattered by a CNT, which is located in free space and exposed to an external plane wave with axial electric field component $E_{0} \exp (i \omega t) ; \omega$ is angular frequency, $z$ and $\rho$ are coordinates of a cylindrical coordinate system with origin being in the nanotube center and axis $z$ directed along the nanotube axis.

Figure 1(a) illustrates the distribution of the intensity enhancement factor $\xi(\rho, z)=$ $|E(\rho, z)|^{2} /\left|E_{0}\right|^{2}$ nearby the right half of a metallic CNT at frequency $1 \mathrm{GHz}$. As follows from figure 1 (a) the value $\xi$ is maximal near the CNT edges; it is about 500 near the nanotube surface and about 10 at a distance of 10-20 nm from the tube axis. The surface charge distribution along the metallic CNT, as well as the intensity enhancement factor distribution nearby the tube, do not depend on the frequency in the quasi-electrostatic regime $(0-100 \mathrm{GHz})$.

Figure 1(b) demonstrates the values of normalized space volume restricted by the surface of constant value $\xi$. As is shown in figure 1(b), the CNT can enhance the field in the volume, which is as much as 100 times larger than the volume occupied by the CNT 
itself. The near-field enhancement effect is expected to provide additional absorption in the surrounding host material and, consequently, modify the effective permittivity of the CNT-based composite medium. In order to clarify this modification, let us first consider a composite material comprising infinitely conductive inclusions. Such inclusions do not absorb electromagnetic energy [7] but they can enhance the field in the near-field zone and provide absorption enhancement in the host medium.

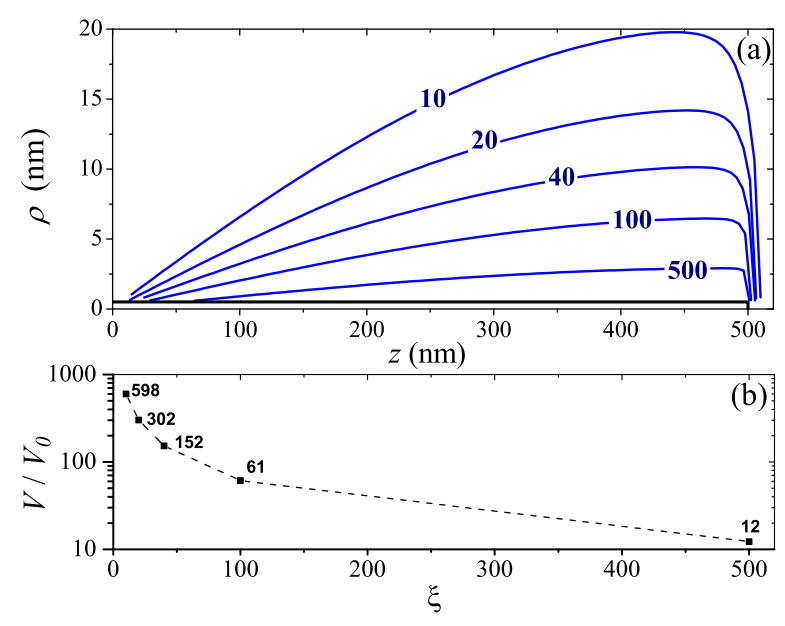

Figure 1. (a) Lines of the calculated constant electric field-intensity enhancement factor $\xi(\rho, z)=\left|E(\rho, z) / E_{0}\right|^{2}$ in the vicinity of the right half of an isolated zigzag $(12,0)$ CNT. $\rho$ is the radial distance from the CNT. The CNT is of length $L=1$ $\mu \mathrm{m}$, and the tube is located along the $\mathrm{z}$ axis, $z \in[-500,500] \mathrm{nm}$, and depicted as the thin horizontal rectangle. (b) The normalized space volume $V$ restricted by the surface of a constant value $\xi$ given in (a) versus $\xi$. $V_{0}$ is the volume occupied by CNT itself. The numbers indicate the value of $V / V_{0}$ in the points of different values $\xi=10,20,40,100,500$.

\subsection{Near-field enhancement effect in the composite medium of infinitely conductive inclusions.}

Let us consider a diluted composite comprising infinitely conductive nanoparticles embedded in a dielectric host with relative complex permittivity $\varepsilon_{h}$. Let $E_{c, h}(r), \varpi_{c, h}$ and $Q_{c, h}$ be the electric field, time and space averaged energy density, and dissipated energy density in the host medium with (subscript "c") and without (subscript "h") the infinitely conductive inclusions, respectively. According to the law of conservation of energy, the space average intensity enhancement factor $\xi_{t}=\varpi_{c} / \varpi_{h}$ equals the space average absorption enhancement factor $\xi_{a}=Q_{c} / Q_{h}$,

$$
\begin{aligned}
\frac{\varpi_{c}}{\varpi_{h}} & =\frac{Q_{c}}{Q_{h}}, \\
Q_{h, c} & =\frac{\pi}{\lambda_{0} \eta_{0} V} \operatorname{Im}\left[\varepsilon_{h}(f)\right] \int_{V}\left|E_{h, c}(\mathrm{r})\right|^{2} \mathrm{dr}^{3}, \\
\varpi_{h, c} & =\frac{\varepsilon_{0}}{2 V} \operatorname{Re}\left[\varepsilon_{h}(f)\right] \int_{V}\left|E_{h, c}(\mathrm{r})\right|^{2} \mathrm{dr}^{3},
\end{aligned}
$$


where $\lambda_{0}$ is free space wavelength, $\eta_{0}=\sqrt{\mu_{0} / \varepsilon_{0}}$, with $\mu_{0}$ being the permeability and $\varepsilon_{0}$ the permittivity of free space. The averaging should be taken over the volume $V$ comprising many $(>100)$ inclusions. The characteristic size of the volume should be much less than the wavelength in the host medium $\left(V^{1 / 3} \ll \lambda_{0} R e \sqrt{\varepsilon_{h}}\right)$. In (2)-(3) the integration volume $V$ does not include the area occupied by inclusions themselves; this approximation can be accepted when the volume fraction of the inclusions is small $\left(<10^{-3}\right)$.

According to the homogenization procedure of effective medium theory, we can replace the realistic composite material with a homogenous medium having the effective permittivity $\varepsilon_{e f f}$ and effective electric field inside $E_{\text {eff }}$. Equation (1) remains true for the homogenous medium for the following parameters:

$$
\begin{aligned}
& Q_{c}=\frac{\pi}{\lambda_{0} \eta_{0} V} \operatorname{Im}\left[\varepsilon_{e f f}(f)\right] \int_{V}\left|E_{e f f}(\mathrm{r})\right|^{2} \mathrm{dr}^{3}, \\
& \varpi_{c}=\frac{\varepsilon_{0}}{2 V} \operatorname{Re}\left[\varepsilon_{e f f}(f)\right] \int_{V}\left|E_{e f f}(\mathrm{r})\right|^{2} \mathrm{dr}^{3},
\end{aligned}
$$

Substitution of $Q_{c}$ and $\varpi_{c}$ from (4) and (5) and $Q_{h}$ and $\varpi_{h}$ from (2) and (3) into (1) leads to the following relation between the imaginary and real parts of the effective permittivity of the composite medium comprising infinitely conductive inclusions,

$$
\frac{\operatorname{Im}\left(\varepsilon_{e f f}\right)}{\operatorname{Im}\left(\varepsilon_{h}\right)}=\frac{\operatorname{Re}\left(\varepsilon_{e f f}\right)}{\operatorname{Re}\left(\varepsilon_{h}\right)},
$$

Equation (6), and the equation that follow from it, (16), are the main theoretical points of this work. This is because, in the quasistatic regime, the field distribution around infinitely conductive nanoparticles does not depend on frequency, so that the ratios in (6) and values $\xi_{t}$ and $\xi_{a}$ are frequency independent. The values $\varepsilon_{h}$ and $\varepsilon_{e f f}$ can be found from measurements and used for justification, via (6), of the near-field enhancement effect in a composite medium. That is, (6) is based on assuming infinitely conductive nanotubes, in which case the only energy absorption mechanism is due to fields in the lossy host medium. Thus, when (6) is seen to be valid in measurements, i.e., when the ratios are frequency independent, we can attribute absorption to be primarily due to absorption in the host (i.e., we can neglect absorption in the nanoparticles themselves). Further, since in the absence of nanoparticles the ratios are identically unity, any ratio value greater than unity indicates absorption enhancement due to the presence of the nanoparticles. This is the main point of the paper, which we next demonstrate both theoretically and experimentally.

\subsection{Theoretical effective permittivity of composite comprising a mixture of high-and low-conductive CNTs}

Absorption enhancement in a CNT-based composite medium occurs via energy dissipation in low-conductive tubes and additional energy dissipation in the host medium due to the near-field enhancement effect of high-conductive CNTs [2]. In this section, to justify the use of (6) for finite-yet-high-conductivity CNTs, we will first analyze the 
contributions of low- and high-conductive nanotubes to the effective permittivity of a CNT-based suspension.

At present, two models have been developed to describe the electromagnetic response of carbon nanotubes from the radiofrequency to the terahertz frequency range. A strict electromagnetic model of a CNT as a hollow cylinder with an infinitely thin wall and effective axial surface conductivity has been proposed in [27]. This model has been applied to calculate the polarizability[13] and antenna parameters [13, 11, 12] of finite length carbon nanotubes. It has also been shown $[28,29]$ that finite-length CNTs can be modeled as a prolate ellipsoidal nanoparticle made of homogeneous anisotropic material. The hollow cylinder model [13] as well as the ellipsoid model [28, 29] have been applied to describe the localized plasmon resonance in CNT thin films in the terahertz range. Both models give identical values of the axial nanonotube polarizability in a frequency range below $10 \mathrm{THz}$ (see figure 2 in [28]). Further, we shall use the ellipsoid model, as it leads to a simple formula for CNT polarizability and the effective permittivity of CNT suspensions.

Let us consider the effective dielectric parameters of a suspension comprising randomly dispersed, randomly oriented CNTs of different lengths and radii in the microwave and radiofrequency ranges. We designate the single index $j$ to identify the type of CNT. Suppose that each CNT of $j$-type is a prolate spheroid made of an orthorhombic material with relative permittivity

$$
\varepsilon_{c n}^{(j)}=1-\left(\omega_{p}^{(j)}\right)^{2} /[\omega(\omega+i \nu)]
$$

in the axial direction, and whose relative permittivity in any direction normal to the axis equals unity [28]. For metallic CNTs,

$$
\omega_{p}^{(j)}=e\left(6 \gamma_{0} b / \varepsilon_{0}\right)^{1 / 2} /\left(R_{j} \pi \hbar\right),
$$

where $\gamma_{0}=3 \mathrm{eV}$ is the overlap integral; $\hbar$ is the reduced Planck constant; $b=0.142 \mathrm{~nm}$ is the interatomic distance in graphene; $R_{j}$ is the CNT radius; $\nu$ is the electron relaxation frequency, which will be supposed to equal $2 \times 10^{13} \mathrm{~s}^{-1}$. [30] Existing experiments at terahertz frequencies [31] and dc [32] show that the conductivity of low-conductive sCNTs is only several orders of magnitude lower than the conductivity of m-CNTs, rather than the many orders of magnitude expected from Drude theory. This can be explained by impurities and atmospheric oxygen doping in CNTs. Therefore, for calculations we shall suppose a plasma frequency $\omega_{p}^{(s)}$ for low-conductive s-CNTs to be $1000^{1 / 2}$ times lower than that for $\mathrm{m}-\mathrm{CNTs}$ with the same radius: $\omega_{p}^{(s)}=10^{-3 / 2} \omega_{p}^{(m)}$, where $\omega_{p}^{(m)}$ can be found from (8).

The relative permittivity of the suspension can be determined from the WatermanTruell formula [33, 34] adapted to elongated ellipsoidal inclusions,

$$
\varepsilon_{e f f}=\varepsilon_{h}\left(1+\sum_{j} \frac{F^{(j)}}{3} \frac{\varepsilon_{c n}^{(j)}-\varepsilon_{h}}{\varepsilon_{h}+N^{(j)}\left(\varepsilon_{c n}^{(j)}-\varepsilon_{h}\right)}\right),
$$

where $F^{j}$ is the volume fraction occupied by the CNTs (conceived as ellipsoids of volume $2 \pi R_{j}^{2} L_{j} / 3 ; L_{j}$ is CNT length); the factor $1 / 3$ in (9) is due to the random orientations 
of the CNTs; $\varepsilon_{h}$ is the relative permittivity of the host medium; the depolarization factor is determined by the parameters of the CNT, $N^{(j)}=4\left(R_{j} / L_{j}\right)^{2}\left[\ln \left(L_{j} / R_{j}\right)-1\right]$. Equation (9) takes into account only the axial component of ellipsoid polarizability, as the transverse component is much smaller and can be omitted for random ellipsoid orientation in the suspension. Also, (9) is true for composites without percolation, that corresponds to stable suspensions without aggregation effects.

Assume, for simplicity, that the suspension comprises CNTs of two different types: low-conductive s-CNTs and high-conductive m-CNTs with parameters identified by indexes "s" and "m", respectively, i.e., $j=$ "s","m". All the tubes have the same radius, length and depolarisation factor $N$. Then, the right side of (9) can be divided in three parts

$$
\varepsilon_{e f f}=\varepsilon_{h}+\varepsilon_{s}+\varepsilon_{m},
$$

where $\varepsilon_{s}$ and $\varepsilon_{m}$ are contributions from s- and m-CNTs, respectively.

For typical m-CNTs of length $0.5-1 \mu \mathrm{m}$ and radius $0.5-1 \mathrm{~nm}$, and host permittivity $\left|\varepsilon_{h}\right| \leq 100$, in the microwave range the conditions

$$
\operatorname{Re}\left[\varepsilon_{c n}^{(m)}\right] \gg \operatorname{Re}\left[\varepsilon_{h}\right], \quad \operatorname{Im}\left[\varepsilon_{c n}^{(m)}\right] \gg \operatorname{Im}\left[\varepsilon_{h}\right]
$$

are fulfilled; below some edge frequency the conditions

$$
\begin{aligned}
& N \operatorname{Re}\left[\varepsilon_{c n}^{(m)}-\varepsilon_{h}\right] \gg \operatorname{Re}\left[\varepsilon_{h}\right], \\
& N \operatorname{Im}\left[\varepsilon_{c n}^{(m)}-\varepsilon_{h}\right] \gg \operatorname{Im}\left[\varepsilon_{h}\right],
\end{aligned}
$$

are also fulfilled. In this case, we can derive the value $\varepsilon_{m}$ from (9) as

$$
\varepsilon_{m} \simeq \frac{F^{(m)} \varepsilon_{h}}{3 N} \text {. }
$$

If the suspension comprises only m-CNTs, then its effective permittivity $\varepsilon_{e f f}^{(m)}$ can be found from (9) and (14),

$$
\varepsilon_{e f f}^{(m)}=\varepsilon_{h}+\varepsilon_{m} \simeq \varepsilon_{h}\left(1+\frac{F^{(m)}}{3 N}\right) .
$$

According to (15) the ratio

$$
\frac{\operatorname{Im}\left[\varepsilon_{\text {ef }}^{(m)}\right]}{\operatorname{Im}\left[\varepsilon_{h}\right]}=\frac{\operatorname{Re}\left[\varepsilon_{\text {eff }}^{(m)}\right]}{\operatorname{Re}\left[\varepsilon_{h}\right]} \simeq 1+\frac{F^{(m)}}{3 N},
$$

does not depend on the frequency and parameters of the host, and can give us information about the volume fraction or aspect ratio of the inclusions.

Equation (16) completely coincides with the condition (6) for the regime of absorption enhancement due to local field enhancement in composites comprising infinitely conductive nanoparticles. At a typical carbon nanotube radius $0.5 \mathrm{~nm}$ and length from 0.1 to $1 \mu \mathrm{m}$, the value $N$ changes from $6.6 \times 10^{-6}$ to $4.3 \times 10^{-4}$. For suspensions comprising such identical metallic tubes at volume fraction $F^{(m)}=10^{-5}$, the right side of (16) can vary in the range from 1.008 to 1.505, and therefore can be observed in experiment. 
For sufficiently long $(2-10 \mu \mathrm{m})$ CNTs the conditions (12) and (13) are not satisfied in the microwave range, though they remain true at lower frequencies. Therefore for observation of the regime described by (6) in the microwave range we should use m-CNTs of less than $1 \mu \mathrm{m}$ in length. Because of localized plasmon resonance phenomena in the range 1-10 THz [28], energy dissipation in finite-length $\mathrm{m}-\mathrm{CNTs}$ is strong. Consequently, the regime described by (6) can not be observed in the terahertz and subterahertz ranges.

Next, we show that energy dissipation in low-conductivity CNTs can be ignored, compared to energy dissipation produced by high-conductivity CNTs in a lossy host. The finite-length effect for s-CNTs is small in the microwave range, so that using conditions $\nu \gg 2 \pi f$ and $\left|\varepsilon_{h}\right| \gg N\left|\varepsilon_{c n}^{(s)}-\varepsilon_{h}\right|$, after substitution (7) into (9) we arrive at

$$
\varepsilon_{s} \simeq \frac{\left(\omega_{p}^{(s)}\right)^{2} F^{(s)}}{3 \nu \omega} i
$$

We have neglected the real part of the permittivity $\operatorname{Re}\left[\varepsilon_{s}\right]$ in (17) as it is much less than both $\operatorname{Re}\left[\varepsilon_{h}\right]$ and $\operatorname{Im}\left[\varepsilon_{s}\right]$, and practically does not contribute to the suspension permittivity (see figure 2(b) below).

According to $(17), \operatorname{Im}\left[\varepsilon_{s}\right]$ vanishes as frequency grows $\operatorname{Im}\left[\varepsilon_{s}(\omega)\right] \sim 1 / \omega$, whilst $\operatorname{Im}\left[\varepsilon_{m}\right]$ remains constant $\operatorname{Im}\left[\varepsilon_{m}(\omega)\right] \simeq$ const. As a result, at $F^{(s)} \simeq F^{(m)}$ and the inequality $\operatorname{Im}\left[\varepsilon_{m}\right]>\operatorname{Im}\left[\varepsilon_{s}\right]$ is true at microwave frequencies. This means that in suspensions comprising mixture of $\mathrm{m}$ - and $\mathrm{s}-\mathrm{CNTs}$ the mechanism of energy dissipation in the host due to local field enhancement by m-CNTs dominates over the mechanism of energy dissipation in s-CNTs. Thus, it is expected that low-conductive s-CNTs provide a negligibly small contribution to the effective permittivity of CNT-based suspension in microwave range, compared to high-conductive m-CNTs.

\section{Numerical results and discussion}

In order to demonstrate the difference between EM interactions of high-conductive mCNTs and low-conductive s-CNTs, using (9) we calculated the normalized value of the relative permittivity (with unit shift) for suspensions comprising only m-CNTs (figure $2(\mathrm{a})$ ) or only s-CNTs (figure 2(b)). As shown in figure 2(a), the effective permittivity of the m-CNT suspension satisfies (6) for infinitely conductive nanoparticles. In figure $2(\mathrm{~b})$, the value $\operatorname{Im}\left[\varepsilon_{\text {eff }}\right]$ for the s-CNT based suspension decreases as frequency grows, in accordance with (17). The strong difference between the EM response of $\mathrm{m}$ - and s-CNTs is caused by the different mechanisms of EM interaction: s-CNTs are slightly polarized and strongly absorb energy by itself, while m-CNTs are strongly polarized and induce a strong near-field that results in absorption enhancement in the surrounding host medium $[2]$.

In order to compare the contributions of semiconducting and metallic parts to the relative effective permittivity of $\mathrm{m}$ - and s-CNT mixtures, from (9) we calculated the value $\varepsilon_{\text {eff }}$ assuming identical semiconducting and metallic CNTs of radius $0.5 \mathrm{~nm}$ and length $1 \mu \mathrm{m}$ with density ratio $2: 1$ and total volume fraction $\sum_{j} F^{(j)}=3.7 \times 10^{-5}$, 


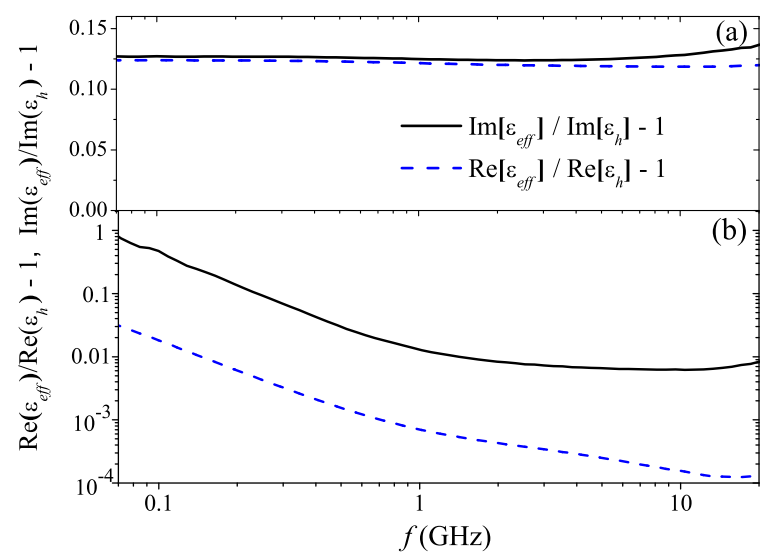

Figure 2. Calculated microwave spectra of the parameters $\operatorname{Im}\left[\varepsilon_{\text {eff }}\right] / \operatorname{Im}\left[\varepsilon_{h}\right]-1$ (black solid line) and $\operatorname{Re}\left[\varepsilon_{e f f}\right] / \operatorname{Re}\left[\varepsilon_{h}\right]-1$ (blue dashed line) for suspensions comprising of identical m-CNTs (a) or s-CNTs (b) of radius $0.5 \mathrm{~nm}$ and length $1 \mu \mathrm{m}$. Nanotube volume fraction (density) was taken as $1.1 \times 10^{-5}(0.033 \mathrm{mg} / \mathrm{ml})$. The value $\varepsilon_{h}$ was taken the same as for 1-Cyclohexyl-2-pyrrolidone from figure 3 .

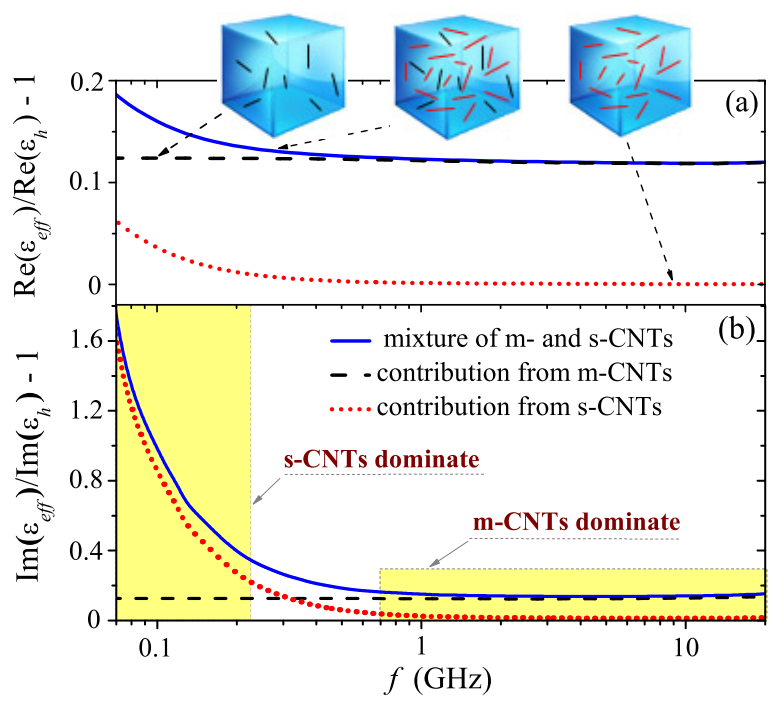

Figure 3. Calculated microwave spectra of the parameters (a) $\operatorname{Re}\left[\varepsilon_{e f f}\right] / \operatorname{Re}\left[\varepsilon_{h}\right]-1$ and (b) $\operatorname{Im}\left[\varepsilon_{e f f}\right] / \operatorname{Im}\left[\varepsilon_{h}\right]-1$ for suspensions comprising of a CNT mixture of s- and m-CNTs with density ratio 2:1 (blue solid line). Nanotube volume fraction (density) was taken as $3.7 \times 10^{-5}(0.1 \mathrm{mg} / \mathrm{ml})$. Contributions of the $\mathrm{m}-$ and $\mathrm{s}-\mathrm{CNT}$ to these parameters are presented by black dashed and red dotted lines, respectively. The value $\varepsilon_{h}$ was taken the same as 1-Cyclohexyl-2-pyrrolidone from figure 5 at $300 \mathrm{~K}$. The yellow area indicates the frequency region where the contribution of m-CNTs or s-CNTs dominates. 
corresponding to a nanotube density $0.1 \mathrm{mg} / \mathrm{ml}$. The microwave spectra of the normalized real and imaginary parts of the effective permittivity with unit shift are presented in figure 3. As shown in figure 3(a), the contribution of the metallic tubes (dashed line) to the real part of the permittivity dominates and does not depend on frequency, according to (6). The same issue is true for the imaginary part of the permittivity at higher frequencies $(>700 \mathrm{MHz}$, see figure $3(\mathrm{~b}))$. At low frequencies $(<400 \mathrm{MHz})$ the contribution of the semiconducting tubes (dotted line) to the value of $\operatorname{Im}\left[\varepsilon_{e f f}\right]$ is lager than the contribution of the metallic tubes, and leads to an increase in the value $\operatorname{Im}\left[\varepsilon_{e f f}\right] / \operatorname{Im}\left[\varepsilon_{h}\right]-1$ with decreasing frequency. Thus, contributions of the metallic and semiconducting tubes to the effective permittivity have different absolute values and frequency dependencies and, therefore, they can be distinguished in experiments.

As a rule, CNTs are produced in bundle form. Also, tube aggregation in suspension leads to the formation of bundles comprising both semiconducting and metallic CNTs. The mechanisms of electromagnetic wave interaction with such bundles and with isolated metallic CNTs are similar. As follows from strict electrodynamic calculations presented in [2] and [35], the radial component of the scattered field near the surface of the metallic CNTs (including into the bundle) is much larger than the incident field due to the high surface charge density at the metallic nanotube ends. This large radial field component provokes the absorption enhancement in the host medium. Semiconducting tubes included in the bundle cannot strongly absorb electromagnetic energy as they are screened by metallic tubes. Indeed, due to the strong depolarizing field in a finite-length metallic CNTs, the total axial field on the surface and near the surface of the tubes is much smaller than the incident field [2]. This screening effect very strongly suppresses energy absorption both in metallic and semiconducting tubes, which form CNT bundles. Thus, we can suppose that the mechanism of absorption enhancement is identical for isolated metallic CNTs and CNT-bundles (even mixed bundles).

The theoretical model used here should be adequate at least up to a nanotube volume fraction of $0.1 \%$ (corresponding to a density of $3 \mathrm{mg} / \mathrm{ml}$ ). However, in order to apply the theory to quantitatively describe experimental results one would need to account for a mixture of nanotubes and bundles taking into account the realistic conductivity of CNTs and their distribution over lengths and diameters. As this information is lacking in our samples, in the following we will not compare quantitatively theory and experiment. Nevertheless, we will demonstrate qualitative agreement, indicating the mechanism of energy absorption.

\section{Experimental details}

For microwave spectroscopy we prepared CNT suspensions based on the host liquid 1-Cyclohexyl-2-pyrrolidone (CHP). As demonstrated in [14] CNTs can form stable solutions in the CHP at CNT densities of up to $3 \mathrm{mg} / \mathrm{ml}$. In experiments we used single-walled CNTs (SWeNT, Inc., SG65i purchased from Sigma-Aldrich) produced by 
chemical vapor deposition on a cobalt-molybdenum catalyst, with an average CNT diameter of 0.7-0.9 nm, purity exceeding 95\% and CNT content of more than 93\%. Most of the tubes are in bundled form. For comparison, we prepared two types of the material, annealed and doped CNTs. To anneal CNTs, some part of CNT material as purchased were placed in vacuum $\left(10^{-4} \mathrm{~Pa}\right)$ for $30 \mathrm{~min}$ at $500{ }^{\circ} \mathrm{C}$. Annealing led to the partial loss of the complexes, which remained on the tube after the purification by acid and caused a doping effect[36]. Annealed CNTs were expected to have bundles of both high-conductive metallic tubes and low-conductive semiconducting tubes. In order to dope, the CNT material was placed in nitric acid (10\%) for 30 hours, followed by removing acid with water-dilution procedure and drying at room temperature for 2 days.

All of the doped CNTs were expected to be high-conductivity. In order to prove that, we prepared CNT thin films using the filtration method, and measured infrared absorbance spectra after annealing and after doping, conducted in the same conditions as mentioned above. Figure 4 shows the infrared absorbance spectra of the film after the doping and annealing treatment. As shown in figure 4, the film absorbance decreases as normalized frequency $\mathrm{f} / \mathrm{c}$ increases from 400 to $4000 \mathrm{~cm}^{-1}$. This behavior is caused by intraband transitions of the quasi-free charges in metallic and doped semiconducting CNTs. As the absorbance of doped CNT film is about 2.6 times higher than that for the annealed film in the range $f / c \in(400,4000) \mathrm{cm}^{-1}$, we may say that the number of high-conductive tubes is about 2.6 times higher for the doped CNT material than for the annealed one.

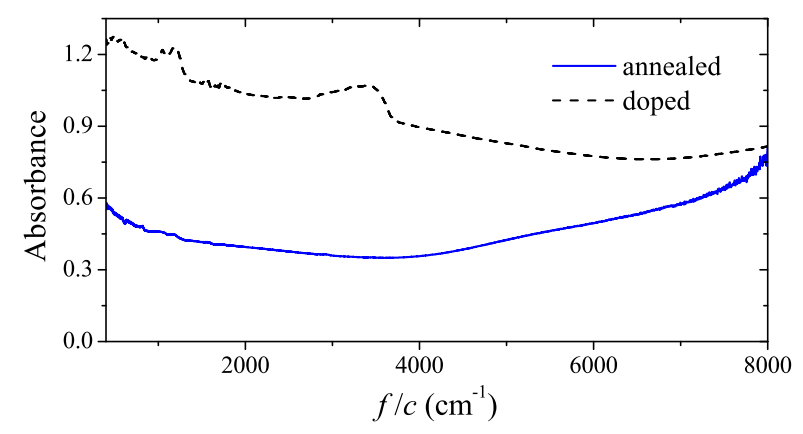

Figure 4. Infrared absorbance spectra of doped and annealed thin CNT film.

To prepare CNT-based suspensions, $2 \mathrm{mg}$ of CNT material (doped or annealed) was suspended in a $2 \mathrm{ml}$ CHP by ultrasonication (Ultrasonic Devise UZDN-2T, Russia, 44 $\mathrm{kHz}$ ) for $4 \mathrm{~h}$ at temperature $20{ }^{\circ} \mathrm{C}$ followed by centrifugation at $900 \mathrm{~g}$ for $1 \mathrm{~h}$. We noticed that CHP degraded in time: the effective permittivity of the CHP sample initially measured and after 10 days are slightly (5\%) different (see Supplemental Materials [26] for details). The CHP was under ultrasonic treatment for 4 hours, and the microwave measurements for all the samples were performed within 15 hours after finishing ultrasonication.

The complex dielectric permittivity was determined from the complex reflection 
coefficient using a lumped admittance method. The complex reflection coefficient was measured using an Agilent E8363B vector network analyzer and Agilent 85070E dielectric probe kit in the $10 \mathrm{MHz}-40 \mathrm{GHz}$ frequency range. The measurements of the suspensions were conducted at different temperatures in the range $T \in(300,330) K$ with a resolution $1 \mathrm{~K}$ using a home made sample heating element.

\section{Experimental results and discussion}

Figure 5(a) shows the microwave spectra of the measured effective permittivity for pure CHP at different temperatures $T \in\{300,310,320,330\} \mathrm{K}$. The spectra have strong dispersion due to the effect of Debye relaxation of the CHP molecules. Variation of the temperature leads to a strong modification of the spectra that gives us a good possibility to change the host permittivity by up to $50 \%$.

Figure 5(b) shows the effective permittivity spectra of the CHP with and without suspended doped and annealed CNTs at $300 \mathrm{~K}$. The contribution of the CNTs to the value of $\varepsilon_{\text {eff }}$ is not strong: about $5 \%$ and $10 \%$ for doped and annealed CNTs, respectively. Hereafter, we shall analyze measured data in the range $f \in(2,20) \mathrm{GHz}$, where the measurement error is less than $2 \%$ (the error is mostly determined by the impact of removing, cleaning, and reinstalling the sample holder after each measurement, see Supplemental Material[26]).

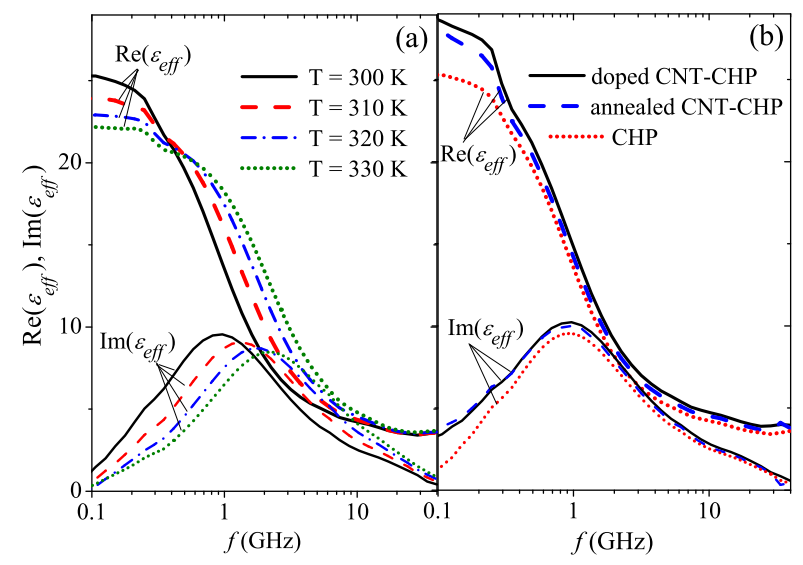

Figure 5. (a) Measured spectra of the relative permittivity of the CHP at different temperatures $T \in\{300,310,320,330\}$ K. (b) Measured spectra of the relative permittivity of the CHP with and without suspended doped and annealed CNTs at $300 \mathrm{~K}$.

Figure 6 shows the measured temperature dependence of the effective permittivity for CNT-CHP suspensions with doped and annealed tubes, and pure CHP at $3.16 \mathrm{GHz}$. The measurements were done during sample heating (up arrow) and cooling (down arrow). As shown in figure $6(\mathrm{a}, \mathrm{b})$, both the imaginary and real parts of the effective permittivity increase by $50 \%$ as temperature increases from 300 to $330 \mathrm{~K}$. There is not strict coincidence between data obtained at the same temperature in the heating and 
cooling regimes. We explain this by the error of our measurements and possibly by the partial aggregation of CNTs which could be occurred at higher temperatures.

As follows from the data in [37], the conductance of the CNT increases by 6 $\%$ as temperature decreases from 290 to $260 \mathrm{~K}$. We expect approximately the same temperature dependence of the CNT conductivity in the range $T \in(300,330) \mathrm{K}$. Although the heating can slightly increase energy dissipation in the CNTs, in accordance with our theoretical estimations, we expect that energy dissipation due to the near-field enhancement effect during the heating remains much higher than ohmic dissipation in the CNTs.

Figure 7 is the principle experimental result of this work, showing the microwave spectra of the values $\operatorname{Re}\left[\varepsilon_{\text {eff }}\right] / \operatorname{Re}\left[\varepsilon_{h}\right]$ and $\operatorname{Im}\left[\varepsilon_{\text {eff }}\right] / \operatorname{Im}\left[\varepsilon_{h}\right]$ found from the measured effective permittivity of the suspensions comprising doped (figure $7(\mathrm{a})$ ) and annealed (figure 7(b)) CNTs at 300, 315, and $330 \mathrm{~K}$. From the scale of the vertical axis, it is clear that the ratios in (6) and (16) are nearly frequency-independent, as predicted, indicating that enhanced absorption due to the presence of highly-conducting CNTs is occurring.
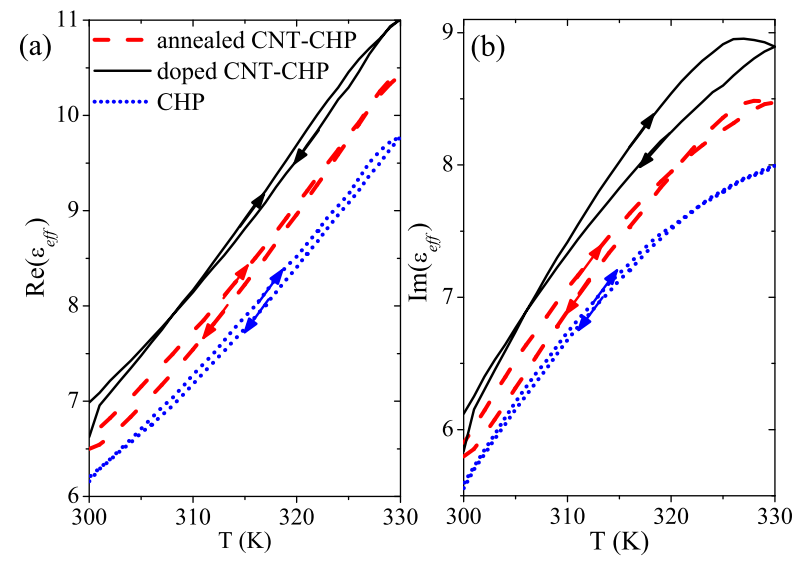

Figure 6. Temperature dependence of the (a) real and (b) imaginary parts of the effective permittivity for CNT-CHP suspensions with doped and annealed tubes and pure CHP at $3.16 \mathrm{GHz}$. Arrows indicate heating and cooling processes.

Furthermore, the enhanced absorption (ratio $>1$ ) near metallic CNTs or CNT bundles [2] is clearly visible in a wide frequency range in figure 7 , where to an accuracy of $30 \%$, the equality $\operatorname{Re}\left[\varepsilon_{e f f}\right] / \operatorname{Re}\left[\varepsilon_{h}\right]-1 \approx \operatorname{Im}\left[\varepsilon_{\text {eff }}\right] / \operatorname{Im}\left[\varepsilon_{h}\right]-1$ is true, in agreement with (6) and (16). The value $1+F /(3 N)$ equals $\approx 1.1$ and $\approx 1.06$ for doped and annealed CNT suspensions, respectively. The deviation, of up to $30 \%$, of the permittivity ratio from being frequency-independent may seem rather poor. However, let us note that the real and imaginary parts of the CHP permittivity (i) decrease about 4 times as the frequency increases from 2 to $20 \mathrm{GHz}$ (ii) vary by up to $50 \%$ as temperature increases from 300 to $330 \mathrm{~K}$. All these strong variations of $\operatorname{Re}\left[\varepsilon_{h}\right]$ and $\operatorname{Im}\left[\varepsilon_{h}\right]$ slightly modify the ratios $\operatorname{Re}\left[\varepsilon_{e f f}\right] / \operatorname{Re}\left[\varepsilon_{h}\right]-1$ and $\operatorname{Im}\left[\varepsilon_{\text {eff }}\right] / \operatorname{Im}\left[\varepsilon_{h}\right]-1$ preserving their equality in a wide frequency range to an accuracy of $30 \%$. This shows that the effective permittivity of CNT-based suspensions satisfies to approximate equality 


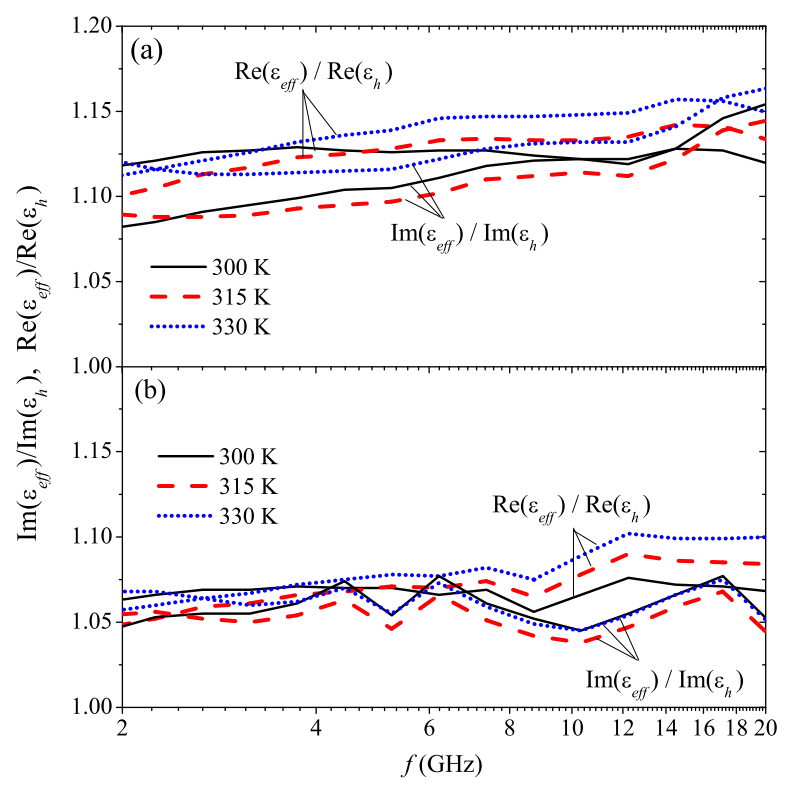

Figure 7. Frequency dependence of the values $\operatorname{Re}\left[\varepsilon_{e f f}\right] / \operatorname{Re}\left[\varepsilon_{h}\right]$ and $\operatorname{Im}\left[\varepsilon_{e f f}\right] / \operatorname{Im}\left[\varepsilon_{h}\right]$ at different temperatures $T \in\{300,315,330\} \mathrm{K}$ for CNT-CHP suspensions comprising (a) doped and (b) annealed tubes.

(16) and consequently has frequency behavior analogous to suspensions comprising infinitely conductive nanoparticles. Simultaneously, this gives an evidence of the nearfield enhancement effect in a CNT suspension according to (6).

Our experimental data demonstrates the unique ability of carbon nanotubes to enhance absorption in a lossy host via a near-field effect, associated with the large radial field near the CNT ends. The effect is due to the enormous axial ratio associated with CNTs. Theoretically, we see that the effect can take place in a volume 100 times larger than the volume occupied by the nanotube itself. This property has not been claimed for any other particle before.

The effective permittivity of a realistic CNT-based solid composite material is greatly affected by aggregation and percolation. Dissipated power in percolated CNTs is comparable or even exceeds the power dissipated in the host material due to the nearfield enhancement effect. At present, it is impossible to obtain solid composite material comprising only individual (non-agglomerated/non-bundled/non-percolated) CNTs at nanotube density more than $1 \mathrm{mg} / \mathrm{ml}$, as the maximal concentration of individual CNT in the solvent, which is used for solid composite preparation, does not usually exceed 1 $\mathrm{mg} / \mathrm{cm}^{3}$. Moreover, the solidification process as a rule leads to partial CNT aggregation. That is why the near-field enhancement regime is difficult to observe in solid CNT-based composite material.

Let us notice that the ratios $\operatorname{Re}\left[\varepsilon_{e f f}\right] / \operatorname{Re}\left[\varepsilon_{h}\right]$ and $\operatorname{Im}\left[\varepsilon_{e f f}\right] / \operatorname{Im}\left[\varepsilon_{h}\right]$ both obtained in experiments for doped tubes (figure 7) and calculated for the model solution (figure 3 ) equal $\approx 1.11$ in the range $2-20 \mathrm{GHz}$. However, in the experiment we used a 
suspension with CNT density of about $1 \mathrm{mg} / \mathrm{ml}$, where most of the CNTs are in bundled form, whereas the theoretical results are for a lower density $(0.1 \mathrm{mg} / \mathrm{ml})$ but assuming individual CNTs. Since the polarizability and absorption cross section of a CNT bundle are very close to those of an individual metallic CNT in the quasi-electrostatic regime (see figure 7 in [2], which shows a comparison of bundles of 2-12 CNTs with individual metallic CNTs), approximately the same microwave response is expected for composites comprising individual and bundled tubes at the same number density. However, the mass densities of the inclusions will differ, and, hence, we compare theory and experiment using different mass densities.

\section{Conclusions}

In conclusion, for suspensions comprising infinitely conductive particles, the approximate equality between the ratio of the imaginary and real parts of the effective permittivity of the suspension and host medium has been derived from the law of conservation of energy. Infinitely conductive particles model well the effect of highlyconducting m-CNTs, and contribute to absorption in the suspension by means of the near-field enhancement effect.

The Waterman-Truell approach, adapted to composites comprising elongated ellipsoidal inclusions, was applied to describe the effective permittivity of CNT-based suspensions in the microwave range. As follows from the model, the contributions from the high-conductive metallic and low-conductive semiconducting CNTs to the suspension permittivity have different absolute values and frequency dependence in some parts of the microwave range, and therefore they can be distinguished from each other. The theoretical calculations predict stronger contribution to the effective permittivity from high-conductive nanoparticles than from low-conductive ones in the microwave range. This leads to appropriate conditions for observation of the near-field enhancement effect.

We demonstrated via experiment that in the frequency range $2-20 \mathrm{GHz}$ and in the temperature range 300-330 K, the effective permittivity of the suspensions comprising either annealed or doped CNTs has frequency behavior, to accuracy of 30\%, analogous to suspensions comprising infinitely conductive nanoparticles. Such behavior originates from the EM response of high-conductive metallic and doped semiconducting CNTs and CNT bundles, and gives experimental evidence of the regime of the absorption enhancement due to the effect of near-field enhancement, predicted for the CNTs in the terahertz $[4,5]$ and radiofrequency [2] ranges.

\section{Acknowledgments}

This research was partially supported by the Belarus Republican Foundation for Fundamental Research (BRFFR) under projects F12MV-004, FP7-612285 CANTOR, FP7-316633 POCAONTAS, H2020-MSCA-RISE-2014 project 644076 CoExAN, and the 
European Social Fund under the Global Grant measure (Lithuanian team).

[1] Reich S, Thomsen C and Maultzsch J 2008 Carbon Nanotubes: Basic Concepts and Physical Properties (Wiley) ISBN 9783527618057 URL https://books . google. by/books?id=nlRWOE6COAsC

[2] Shuba M V, Slepyan G Y, Maksimenko S A and Hanson G W 2010 J. Appl. Phys. 108114302 URL http://scitation.aip.org/content/aip/journal/jap/108/11/10.1063/1.3516480

[3] Shuba M, Paddubskaya A, Plyushch A, Kuzhir P, Slepyan G Y, Maksimenko S, Ksenevich V, Buka P, Seliuta D, Kasalynas I, Macutkevic J, Valusis G, Thomsen C and Lakhtakia A 2012 Phys. Rev. B 85(16) 165435 URL http://link.aps.org/doi/10.1103/PhysRevB.85.165435

[4] Shuba M V, Maksimenko S A and Slepyan G Y 2009 J. Comput. Theor. Nanosci. 6 2016-2023 URL http://www. ingentaconnect. com/content/asp/jctn/2009/000000006/00000009/art00006

[5] Nakanishi $\mathrm{T}$ and Ando T 2009 J. Phys. Soc. Jpn. 78 114708- ISSN 0031-9015 URL http://dx.doi.org/10.1143/JPSJ.78.114708

[6] Dovbeshko G, Gnatyuk O, Fesenko O, Rynder A and Posudievsky O 2012 Journal of Nanophotonics 6 061711-061711 ISSN 1934-2608 URL http://dx.doi.org/10.1117/1. JNP.6.061711

[7] Hanson G W and Patch S K 2009 J. Appl. Phys. $106 \quad 054309$ URL http://scitation.aip.org/content/aip/journal/jap/106/5/10.1063/1.3204653

[8] Hartmann R R, Kono J and Portnoi M E 2014 Nanotechnology 25322001 URL http: //stacks . iop.org/0957-4484/25/i=32/a=322001

[9] Yu Z and Burke P J 2005 Nano Lett. 5 1403-1406 URL http://dx.doi.org/10.1021/n1050738k

[10] Jensen S A, Ulbricht R, Narita A, Feng X, Mllen K, Hertel T, Turchinovich D and Bonn M 2013 Nano Lett. 13 5925-5930 URL http://dx.doi.org/10.1021/nl402978s

[11] Hanson G 2005 IEEE Trans. Antennas Propag. 53 3426-3435 ISSN 0018-926X URL http://dx.doi.org/10.1109/TAP.2005.858865

[12] Burke P J, Li S and Yu Z 2006 IEEE Trans. Nanotechnol. 5 314-334 ISSN 1536-125X URL http://dx.doi.org/10.1109/TNANO.2006.877430

[13] Slepyan G, Shuba M, Maksimenko S and Lakhtakia A 2006 Phys. Rev. B 73(19) 195416 URL http://link.aps.org/doi/10.1103/PhysRevB.73.195416

[14] Bergin S D, Sun Z, Streich P, Hamilton J and Coleman J N 2010 J. Phys. Chem. C 114 231-237 URL http://dx.doi.org/10.1021/jp908923m

[15] Mashal A, Sitharaman B, Li X, Avti P, Sahakian A, Booske J and Hagness S 2010 IEEE Trans. Biomed. Eng. 57 1831-1834 ISSN 0018-9294 URL http://dx.doi.org/10.1109/TBME. 2010.2042597

[16] Gach H M and Nair T 2010 Bioelectromagnetics 31 582-588 ISSN 1521-186X URL http://dx.doi.org/10.1002/bem. 20595

[17] Nair T, Symanowski J T and Gach H M 2012 Bioelectromagnetics 33 134-146 ISSN 1521-186X URL http://dx.doi.org/10.1002/bem. 20689

[18] Xie S X, Gao F, Patel S C, Booske J H, Hagness S C and Sitharaman B 2013 Appl. Phys. Lett. 103133114 URL http://scitation.aip.org/content/aip/journal/apl/103/13/10.1063/1.4823541

[19] Bourdiol F, Dubuc D, Grenier K, Mouchet F, Gauthier L and Flahaut E 2015 Carbon $\mathbf{8 1} \quad 535-545$ ISSN 0008-6223 URL http://www.sciencedirect.com/science/article/pii/S0008622314009518

[20] Corr S, Raoof M, Cisneros B, Orbaek A, Cheney M, Law J, Lara N, Barron A, Wilson L and Curley S 20158 2859-2870- ISSN 1998-0124 URL http://dx.doi.org/10.1007/s12274-015-0791-1

[21] Vichchulada P, Cauble M A, Abdi E A, Obi E I, Zhang Q and Lay M D 2010 J. Phys. Chem. C 114 12490-12495 URL http://dx.doi.org/10.1021/jp104102t

[22] See, for example, the parameters of electronically separated single wall carbon nanotubes at http://www.nanointegris.com

[23] Blackburn J L, Barnes T M, Beard M C, Kim Y H, Tenent R C, McDonald T J, To B, Coutts T J and Heben M J 2008 ACS Nano 2 1266-1274 ISSN 1936-0851 URL http://dx.doi.org/10.1021/nn800200d 
[24] Collins P G, Bradley K, Ishigami M and Zettl A 2000 Science 287 1801-1804 URL http://www. sciencemag. org/content/287/5459/1801

[25] Zhang Q, Hroz E H, Jin Z, Ren L, Wang X, Arvidson R S, Lttge A and Kono J 2013 Nano Lett. 13 5991-5996 ISSN 1530-6984 URL http://pubs.acs.org/doi/abs/10.1021/nl403175g

[26] See supplemental material for the theory of electromagnetic wave scattering by finite-length SWCNT and details of SWCNT-based suspension preparation.

[27] Slepyan G Y, Maksimenko S A, Lakhtakia A, Yevtushenko O and Gusakov A V 1999 Phys. Rev. B 60(24) 17136-17149 URL http://link.aps.org/doi/10.1103/PhysRevB.60.17136

[28] Slepyan G, Shuba M, Maksimenko S, Thomsen C and Lakhtakia A 2010 Phys. Rev. B 81(20) 205423 URL http://link.aps.org/doi/10.1103/PhysRevB.81.205423

[29] Mikki S and Kishk A 2009 IEEE Trans. Antennas Propag. 57 1412-1419 ISSN 0018-926X

[30] Shuba M V, Slepyan G Y, Maksimenko S A and Hanson G W 2011 Nanosci. Nanotechnol. Lett. 3 885-888 URL http://dx.doi.org/10.1166/nnl.2011.1257

[31] Beard M C, Blackburn J L and Heben M J 2008 Nano Lett. 8 4238-4242 URL http://dx.doi.org/10.1021/nl801913y

[32] Zhou X, Park J Y, Huang S, Liu J and McEuen P 2005 Phys. Rev. Lett. 95(14) 146805 URL http://link.aps.org/doi/10.1103/PhysRevLett.95.146805

[33] Waterman P C and Truell R 1961 Journal of Mathematical Physics 2 512-537 URL http://scitation.aip.org/content/aip/journal/jmp/2/4/10.1063/1.1703737

[34] Lakhtakia A 1993 International Journal of Electronics 75 1243-1249 ISSN 0020-7217 URL http://dx.doi.org/10.1080/00207219308907198

[35] Nemilentsau A, Shuba M, Slepyan G, Kuzhir P, Maksimenko S, D'yachkov P and Lakhtakia A 2010 Phys. Rev. B 82(23) 235424 URL http://link.aps.org/doi/10.1103/PhysRevB.82.235424

[36] Itkis M E, Niyogi S, Meng $M$ E, Hamon M A, Hu H, and Haddon* R C 2002 Nano Letters 2 155-159 (Preprint http://dx.doi.org/10.1021/nl0156639) URL http://dx.doi.org/10.1021/nl0156639

[37] Zhou C, Kong J and Dai H 2000 Phys. Rev. Lett. 84(24) 5604-5607 URL http://link.aps.org/doi/10.1103/PhysRevLett.84.5604 